\title{
Updated phase coherent timing solution of the isolated neutron star RX J0720.4-3125 using recent XMM-Newton and Chandra observations ${ }^{\star}$
}

\author{
M. M. Hohle ${ }^{1,2}$, F. Haberl ${ }^{1}$, J. Vink ${ }^{3}$, R. Turolla ${ }^{4,5}$, S. Zane ${ }^{5}$, C. P. de Vries ${ }^{6}$, and M. Méndez \\ 1 Max-Planck-Institut für extraterrestrische Physik, Giessenbachstraße, 85741 Garching, Germany \\ e-mail: mhohle@astro.uni-jena.de \\ 2 Astrophysikalisches Institut und Universitäts-Sternwarte Jena, Schillergässchen 2-3, 07745 Jena, Germany \\ 3 University Utrecht, PO Box 80000, 3508 TA Utrecht, The Netherlands \\ ${ }^{4}$ Department of Physics, University of Padua, via Marzolo 8, 35131 Padova, Italy \\ 5 Mullard Space Science Laboratory, University College London, Holmbury St. Mary, Dorking, Surrey, RH5, 6NT, UK \\ 6 SRON, Netherlands Institute of Space Research, Sorbonnelaan 2, 3584 CA Utrecht, The Netherlands \\ 7 Kapteyn Astronomical Institute, University of Groningen, PO Box 800, 9700 AV Groningen, The Netherlands
}

Received 13 November 2009 / Accepted 6 July 2010

\section{ABSTRACT}

\begin{abstract}
Aims. Since the last phase coherent timing solution of the nearby radio-quiet isolated neutron star RX J0720.4-3125 six new XMM-Newton and three Chandra observations were carried out. The phase coherent timing solutions from previous authors were performed without restricting to a fixed energy band. However, we recently showed that the phase residuals are energy dependent, and thus phase coherent solutions must be computed referring always to the same energy band.

Methods. We updated the phase coherent timing solution for RXJ0720.4-3125 by including the recent XMM-Newton EPIC-pn, MOS1, MOS2 and Chandra ACIS data in the energy range 400-1000 eV. Altogether these observations cover a time span of almost 10 yrs. A further timing solution was obtained including the ROSAT pointed data. In this case, observations cover a time span of $\approx 16$ yrs. To illustrate the timing differences between the soft band $(120-400 \mathrm{eV})$ and the hard band $(400-1000 \mathrm{eV})$ a timing solution for the soft band is also presented and the results are verified using a $Z_{n}^{2}$ test.

Results. In contrast to previous work, we obtain almost identical solutions whether or not we include the ROSAT or Chandra data. Thanks to the restriction to the hard band, the data points from EPIC-pn are in better agreement with those from MOS1, MOS2 and Chandra than in previous works. In general the phase residuals are still large and vary with time. In particular, the latest XMM-Newton and Chandra data show that the phase residuals have attained relatively large and negative values. Using this and previous timing solutions, the residuals indicate a cyclic behaviour with a period $\approx 7-9$ yrs if the variations follow a sinusoid, or twice this value in case the residuals are modulated by an abs(sine) probably approaching a new minimum around MJD $=55240$ days (February 2010). As an alternative interpretation, the phase residuals can be fitted with a glitch that occured around MJD $=53000$ days.
\end{abstract}

Key words. stars: individual: RX J0720.4-3125 - stars: neutron - stars: magnetic field - X-rays: stars

\section{Introduction}

Since the discovery of the first isolated radio-quiet neutron star RX J1856.4-3754 (Walter et al. 1996) in the ROSAT All-Sky Survey only six more soft X-ray sources with similar properties were identified. They are often referred to as the "Magnificent Seven" (hereafter M 7; two more objects, which may be M 7-like were found by Rutledge et al. 2008; Pires et al. 2009). Unlike other isolated neutron stars the M 7 exhibit pure thermal (black body) emission (with absorption features in some cases) with temperatures $\leq 100 \mathrm{eV}$ (see Haberl 2007). They are connected to faint blue optical counterparts (see Kaplan 2008, for a review) with $m_{\mathrm{B}} \approx 25-28$. Although these objects are dim in the optical bands, the optical fluxes are $\approx 5-10$ times larger than expected by extrapolating the black body X-ray spectrum at low energies

^ Based on observations with XMM-Newton, an ESA Science Mission with instruments and contributions directly funded by ESA Member states and the USA (NASA). (for RBS 1774 the optical excess is a factor of $\approx 30$, see Zane et al. 2008).

A definite explanation of the emission properties of the M7 has not been put forward as yet. Their nearly Planckian spectrum at X-ray energies, in fact, appears difficult to reconcile with the predictions of standard atmospheric models. It has been suggested that their surface layers are in a condensed rather than gaseous state. The phase transition is expected to occur for low temperatures $\left(T \lesssim 10^{6} \mathrm{~K}\right)$ and high magnetic field strengths $\left(B \gtrsim 10^{13} \mathrm{G}\right.$; see Turolla et al. 2004, and references therein and Medin \& Lai 2006a,b, 2007). If a thin hydrogen atmosphere covers the star, condensed surface models may also explain the optical excess (Zane et al. 2007, 2004; Ho et al. 2007).

Among the M7, RX J0720.4-3125 (discovered by Haberl et al. 1997 and identified with a faint blue optical star in Motch \& Haberl 1998; Kulkarni \& van Kerkwijk 1998; Kaplan et al. 2003; Eisenbeiss et al. 2010) has a unique place inasmuch it shows significant variations in its surface temperature, in the equivalent width of an absorption feature seen in the X-ray spectrum and in 
the size of the emitting area (see de Vries et al. 2004; Haberl 2007; Hohle et al. 2009, hereafter H09).

The constant spin down $\dot{P}$ of RX J0720.4-3125 was first estimated to be $\approx 10^{-14} \mathrm{~s} / \mathrm{s}$ in Zane et al. (2002) and an updated timing solution using further observations was presented in Cropper et al. (2004). The phase coherent timing solutions in Kaplan \& van Kerkwijk (2005) and van Kerkwijk et al. (2007, hereafter KvK05 and vK07, respectively), have been computed by including either data from ROSAT, Chandra and XMM-Newton ("all data" solution), or Chandra data only and all data except ROSAT (see vK07). Applying a constant spin-down model leads to large phase residuals, therefore, vK07 included a glitch event in one of their timing solutions which significantly reduced the phase residuals.

These data sets are from various instruments with different energy responses and in different data acquisition modes. However, Cropper et al. (2001) found a hardness ratio variation and a phase shift between the flux and the hardness ratio (both folded into the $8.391 \mathrm{~s}$ pulse period) using XMM-Newton data. Later, de Vries et al. (2004) showed that the energydependent change in the pulse profile is accompanied by a longterm change of the X-ray spectrum and proposed precession as a possible explanation. Haberl et al. (2004) confirmed the dependence of the pulse profiles on the energy and reported a phase lag between soft (120-400 eV) and hard (400-1000 eV) photons. A long term period of $\approx 7$ yrs was found in the spectral variations and the phase residuals, supporting the precession model (Haberl et al. 2006). The phase lag exhibits a long term evolution, its presence appears not to depend on the template used to fit the X-ray pulse profile and it changed sign around MJD = 52800 days (H09). This behaviour has to be taken into account and therefore requires timing solutions for both bands separately. Since the XMM-Newton MOS and the Chandra HRC and ACIS detectors are less sensitive in the soft band, we focus on a phase coherent timing solution for the hard band only. Since the last phase coherent timing solution in vK07, six new XMM-Newton and three new Chandra observations were performed, thus an updated timing solution including the new data is required to distinguish between a periodic trend or a single glitch event.

\section{Observations and data reduction}

We use EPIC (pn, Strüder et al. 2001, MOS1 and MOS2, Turner et al. 2001) data from all 14 XMM-Newton observations performed between May 2000 and November 2007 (for a summary of the instrumental setups see H09) and include the most recent XMM-Newton observations of March 2009 (rev. 1700, performed in full frame mode with the thin filter for pn and both MOS) and September 2009 (rev. 1792, performed in full frame mode with the thin filter for pn and in small window mode with thin filter for both MOS). We analysed the XMM-Newton data with the standard XMM-Newton science analysis system (SAS) version 7.1.0. applying barycentric correction. We use single and double events for both MOS and the pn detector, that are collected within a circular region of 30" (pn) and 8" (MOS) radius. The observations of March and September 2009 were analysed with SAS version 9.0.0, but following the same procedure as for the other observations. All observations are filtered using the GTI files that are generated during the standard data reduction process. The XMM-Newton observation of March 2009 was strongly affected by flares and the GTI files are not sufficient enough to filter out all time intervals with contaminated counts, i.e. for pn only the second half of the exposure time could be used $\left(10.8 \mathrm{ks}\right.$ with $\approx 30 \times 10^{3}$ photons in the hard band), while the MOS data are much less affected. This cut of the pn data is justified posteriori, since the results (periods, phase residuals etc.) for pn and MOS1 \& MOS2 are in good agreement, as it will be seen later.

The Chandra data were analysed with CIAO 4.1. For best corrections of the read-out times and dither in the Advanced CCD Imaging Spectrometer (ACIS, Garmire et al. 2003) Continuous Clocking (CC) data, we first checked that the observations were processed with the Standard Data Processing (SDP) version DS 7.6.3. or later. The coordinate accuracy for all ACIS-CC observations is better than $0.5^{\prime \prime}$, i.e. one pixel. We applied the Charge Transfer Inefficiency (CTI) correction and use the source photons from a rectangular region of $1^{\prime \prime} \times 1^{\prime \prime}$ size covering the brightest pixels (located on chip seven for all ACIS-CC observations). For High-Resolution Camera (HRC, Kraft et al. 1997) data we used photons from zeroth order (circular region of $2^{\prime \prime}$ radius) and both first orders within the standard LETG spectral extraction windows (but limited to 10-60 $\AA$ and the brightest $1^{\prime \prime}$ pixel stripes only). Finally we applied the barycentric correction using axbary for the HRC and ACIS-CC data.

The ROSAT data were taken from Cropper et al. (2004) whom we refer to for details of the observations and the data reduction.

Assuming that no significant variations in the period of RX J0720.4-3125 occur within a few days, we merged several close Chandra observations with the same instrumental setup to reduce the scatter in the phase folded light curves. After this merging we obtain 70 data sets: 16 from XMM-Newton EPIC-pn and 36 from EPIC-MOS1/MOS2 (the XMM-Newton observations rev. 0622 and 0711 from May and October 2003, respectively, have four MOS data sets each), 6 ROSAT data sets (pointings only) and 12 (out of 29) data sets from Chandra observations. All observations are listed in Table 1, first column.

\section{The selection of the hard band $(400-1000 \mathrm{eV})$}

In previous work (Haberl et al. 2004, 2006, and H09) a variable phase lag between soft (120-400 eV) and hard (400-1000 eV) photons of RXJ0720.4-3125 was reported and discussed. Depending on the different detectors and acquisition modes, the data contains different fractions of photons from the two bands that lead to a systematic scatter in the phase residuals. This phenomenon has to be taken into account and requires a different treatment as for the other M 7 if an adequate timing solution has to be found.

In this section we first discuss the cross calibration issues for the different instruments used for the observations of RX J0720.4-3125, then derive an empirical energy band that minimises the scatter in the residuals and discuss the results.

In Haberl et al. (2004, 2006) the photons were divided into a soft (120-400 eV) and a hard band (400-1000 eV) for the EPIC-pn detector to illustrate the phase shift between these two bands. The effective area of the MOS detectors deviates much from that of the pn detector (particularly in the soft band) and the spectral resolution of the MOS detectors is $\sim 100 \mathrm{eV}$ in the soft band (Turner et al. 2001). This resolution is much worse than for gratings of XMM-Newton or Chandra. In addition, the CCD detectors suffer under the contamination of hard photons in the soft band due to redistribution. The MOS and pn observations were executed in different filters: thin, medium and thick. The thick filter significantly suppresses the penetration of soft photons compared to e.g. the thin filter, that also influences the timing properties. 


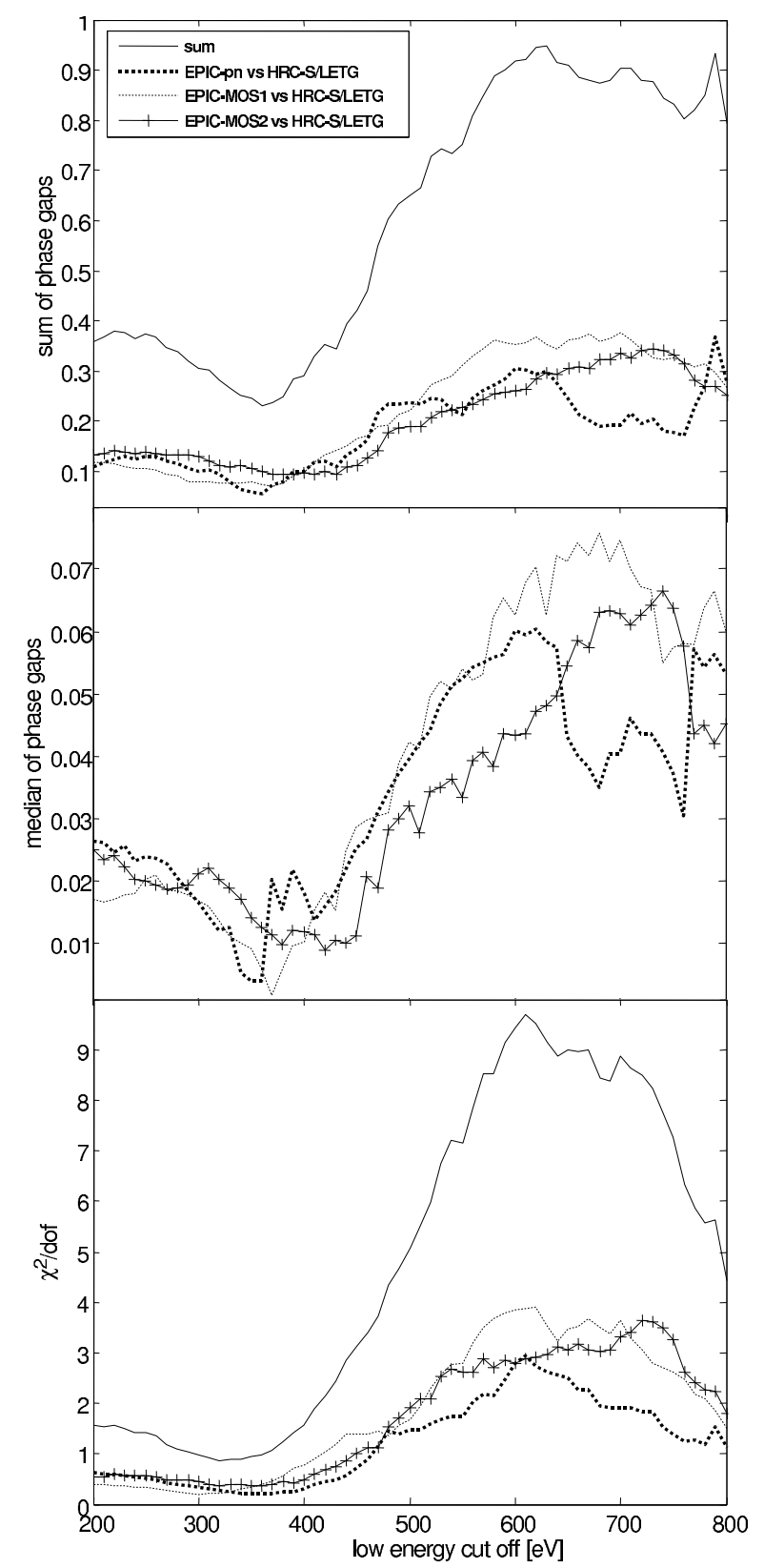

Fig. 1. The accordance of the phase residuals of the hard band (cut off energy-1000 eV) for XMM-Newton EPIC-pn, EPIC-MOS1 \& MOS2 and Chandra HRC of RX J0720.4-3125 for different values of the energy band cut. Data sets taken with different observatories were selected in such a way that they are as close in time as possible. The upper panel shows the sum of the absolute values of the phase differences (phase gaps) derived from the different instruments with respect to each other, while the middle panel shows the median of these phase gaps and the corresponding $\chi^{2} /$ d.o.f. is presented in the lower panel.

The phase residuals from the XMM-Newton observations should be comparable to those of the Chandra observations, taking different energy response, spectral resolution and the phase shift between hard and soft photons into account (softer photons are less prominent in the MOS, HRC and ACIS data than in the pn, influencing the residuals as shown in H09, Fig. 1 and Haberl 2007 Fig. 7). The HRC observations have a low number of photons and the energy information of the photons in the zeroth order is lost. Therefore we included all photons in the HRC data. If we would exclude the zeroth order photons, $\sim 40 \%$ of the counts were lost and if only the hard band (first orders) would be used, $\sim 80 \%$ of the counts were lost, resulting too low statistics. For similar reasons (low statistics, low energy resolution) we also used ROSAT data from a wider energy band: channels 10 to $90(100-900 \mathrm{eV})$ for the position sensitive proportional counter (PSPC) and channels one to eight for the high resolution imager (HRI, David et al. 1996).

The energy band minimising the residuals can be found using those Chandra observations performed close to XMM-Newton observations: The HRC observation 5582 (June 1,2005$)$ is close to the XMM-Newton observation rev. 0986 (April 28, 2005), the HRC observations $6369 \& 7177$ (Oct. 8/9, 2005) are close to the XMM-Newton observation rev. 1060 (Sep. 23, 2005), the HRC observations 7243-7245 (Dec. 14-17, 2005) are close to the XMM-Newton observation rev. 1086 (Nov. 11/12, 2005), the HRC observations $10861 \&$ 10700 (Jan. 20 \& Feb. 14, 2009) are close to the XMM-Newton observation rev. 1700 (Mar. 21, 2009) and the HRC observation 10701 (Sep. 11, 2009) is close to the XMM-Newton observation rev. 1792 (Sep. 22, 2009) see also Table 1, Cols. 6 and 7. Unfortunately no ACIS observation is close in time to these observations.

Assuming that the timing properties of RX J0720.4-3125 do not change significantly within a time span of a month, we compare the timing residuals (using the "all data" timing solution in vK07) of these observations directly changing the band cut from $200 \mathrm{eV}$ to $800 \mathrm{eV}$ in steps of $10 \mathrm{eV}$. For example, if the band cut is at $600 \mathrm{eV}$, we have two bands of 120-600 eV and 600-1000 eV. Due to the limited energy resolution, these band cuts could not be applied for the HRC observations.

We then calculate the phase residuals by fitting a sine for the phase folded light curves (see vK07) in 12 phase bins for pn, MOS1 and MOS2 and HRC for each band cut and calculate the sum of the absolute values and the median of the phase gaps of the phase residuals of the harder band from the different detectors. Having the phase residuals and their errors, we also calculate $\chi^{2} /$ d.o.f. showing the degree of accordance of the results from the different detectors. In all three cases, we derive a minimum (i.e. best agreement) if we use the band cut between $300 \mathrm{eV}$ and $400 \mathrm{eV}$ (for consistency with previous work, Haberl et al. 2004, 2006, and H09, we set the cut between the two bands at $400 \mathrm{eV}$ ), i.e. having two bands of $120-400 \mathrm{eV}$ and 400-1000 eV and using the harder band (see Fig. 1). For band cuts of lower energies the different detector responses for the soft photons cause larger phase gaps, because of the phase shift between hard and soft photons and the rough energy resolution of the EPIC detectors, while for band cuts at higher energies the number of photons decreases and the statistics worsens.

If the same is done, but keeping the soft band photons (see Fig. 2), the best agreement of the different instruments is achieved if no band cut is applied. But even the lowest $\chi^{2} /$ d.o.f. value is more than two times larger as in the case when photons between 400-1000 eV are used.

Apart from the intrinsic properties of RX J0720.4-3125 (the spectrum did not change much since rev. 0986, see H09), the choice of this band cut for the hard photons at least reduces instrumental discrepancies of the different detectors.

\section{Timing procedure}

For comparison with the results of KvK05 (obtained using the $Z_{n}^{2}$ method, Buccheri et al. 1983), we start taking as reference period that from the ACIS-CC observations 4666-4669 combined with the HRC observation 5305, covering a total time span of 


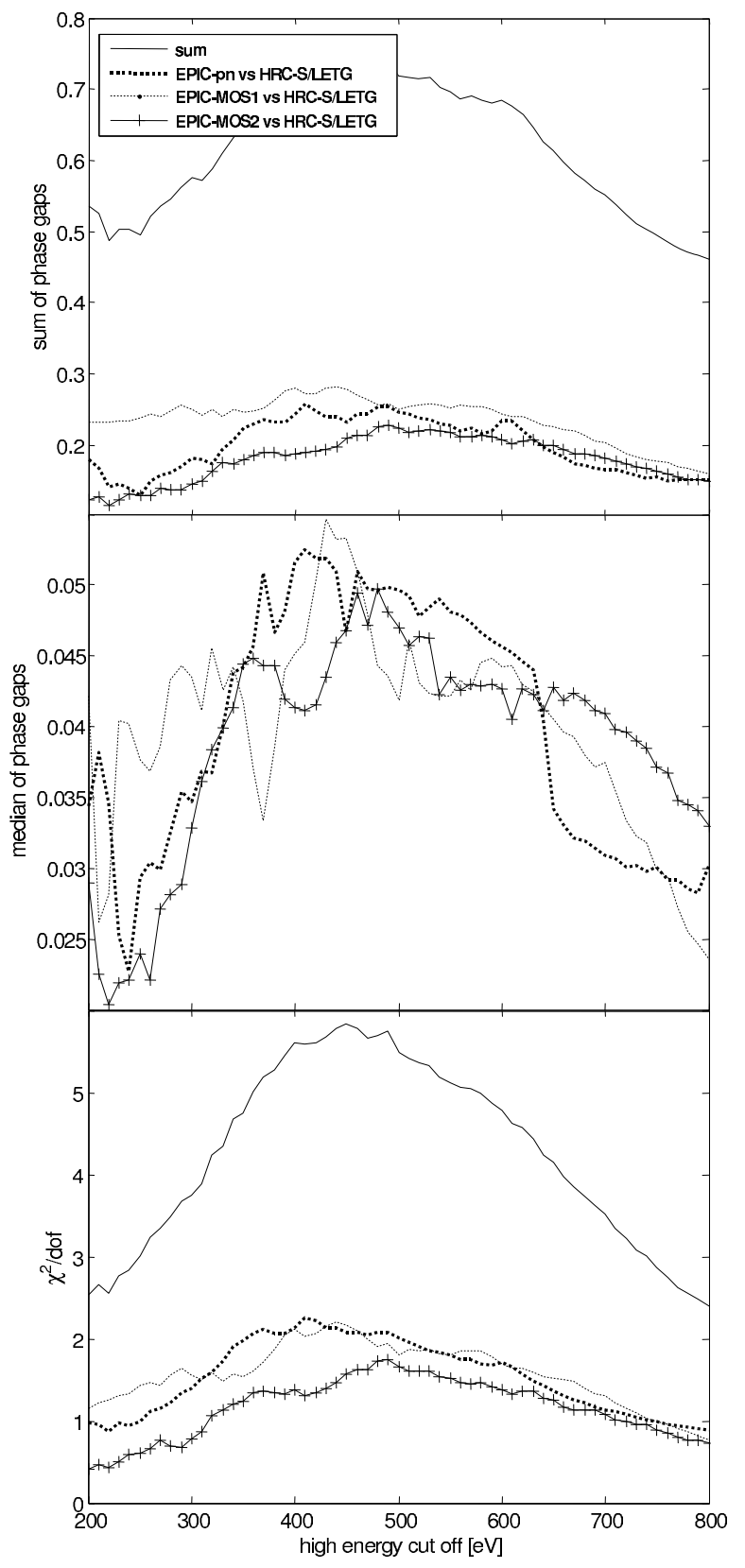

Fig. 2. The same as in Fig. 1, but using the soft band (120 eV-cut off energy) for the analysis.

52 days. We determine the period from the peak in the $Z_{1}^{2}$ periodogram (from now on always $n=1$ ) and estimated the $1 \sigma$ errors following Kaplan et al. (2002) using the equation derived in Ransom et al. (2002). The period we have taken corresponds to the center of the $1 \sigma$ region in the periodogram (since the peak is symmetric close to its maximum).

Using this dataset produces aliases in the periodogram caused by the time gaps between the observations. We obtain three peaks with $Z_{1}^{2} \approx 140: P_{1}=8.39036664(53) \mathrm{s}, P_{2}=$ $8.39111600(50) \mathrm{s}$ and $P_{3}=8.39188114(53) \mathrm{s} . P_{2}$ yields formally the largest $Z_{1}^{2}$ value and is consistent with the reference period $P=8.39111590(50)$ s from KvK05 (numbers in parentheses indicate $1 \sigma$ errors using the error estimation in Ransom et al. 2002). During the timing procedure we found $P_{2}$ being the correct period since starting with $P_{1}$ or $P_{3}$ as initial period, the procedure does not converge.
As a first step we determined the period of each observation separately using the $Z_{1}^{2}$ method. The period of the Chandra HRC observation 7251 shows a large discrepancy with respect to the others, probably caused by the small number of photons giving not enough statistics for a reliable period determination. Therefore we excluded this observation from our analysis. The periods obtained from the ROSAT data with a low count number differ sensibly from the reference period and deviate significantly from those periods listed in Cropper et al. (2004) and are highly uncertain, thus are also excluded for the first phase coherent timing solution. No periodic signal was found in the ROSAT all sky survey, i.e. this observation is not used at all and not listed in Table 1.

Most of the individual periods with more than $10^{4}$ photons are consistent with the single spin-down model within $1 \sigma$ errors (Ransom et al. 2002) and all of them are consistent within $2 \sigma$ errors, both for the hard and the soft band. Note that the periods for Chandra HRC and ROSAT data are the same for both bands since we applied no energy selection for these observations, as mentioned before. All individual periods are listed in Table 2, Cols. 4 and 5 .

Our timing procedure follows that in KvK05 with the difference that we calculate the times of arrival (TOA, the time of maximum light closest to the middle of the observation, see KvK05) from the included observations during the iterations of the timing procedure. The errors of $\dot{f}$ and $P$ are evaluated after each step of the iteration: in the case of $\dot{f}$ the error is calculated from the different $\dot{f}$ values satisfying the condition to minimise the phase residuals within their $1 \sigma$ errors. This restricts the possible values for $\dot{f}$ if more observations are added, i.e. the error on $\dot{f}$ decreases with growing time $T$ from the reference point. The error of $P, e P$, automatically decreases with growing time span $T$ following $e P \sim 1 / T$ (e.g. see the general approach in Kovacs 1981).

In KvK05, vK07 and H09 the phase binned light curves were fitted with a sinusoid to derive the phase of the maximum, although the pulse profiles are not always best represented by a sine function (Haberl et al. 2004). We fitted the light curves using a combination of three harmonics of the Fourier series ${ }^{1}$ as follows: The Fourier series for the light curves is given by:

$F(\phi)=\sum_{k=0}^{\infty} A_{k} \sin (k \phi)+B_{k} \cos (k \phi)$

where $k=0$ corresponds to the constant offset. We choose to fit the light curves with the sum of three terms, not to introduce too many degrees of freedom. To simplify the notation, in the following we denote each term just as sink or cosk, e.g. sin1 stands for $A_{1} \sin (1 \phi), \cos 1$ for $B_{1} \cos (1 \phi)$. Since the contribution of $\cos 1$ turned out to be negligible (since we forced the phase to be zero at the reference time, and a cosine term would imply a phase shift), our sum starts always with sin1, while the remaining two terms can be $\sin 2+\sin 3, \cos 2+\sin 3$ or $\cos 2+\sin 2$, and so on. To keep the number of free parameters small, we do not use terms with $k>3$ and use only three terms in total. The combination that fits the individual light curve with the lowest value of $\chi^{2} /$ d.o.f. was used for the phase determination. This leads to an improvement of the $\chi^{2} /$ d.o.f. for the light curves (see example in Fig. 3) and to a better determination of the phase shift.

1 The light curves are fitted using the MATLAB ${ }^{\circledR}$ (version R2008a) internal function fit with default adjustments. Errors derived from this function and the errors derived from the results of fit are scaled to $\sqrt{\chi^{2} / \text { d.o.f. }}$ 


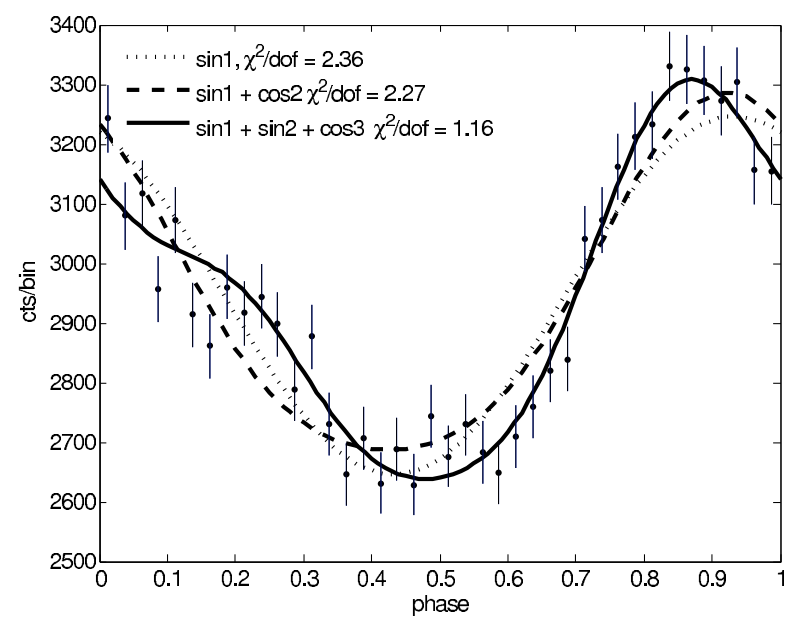

Fig. 3. Phase binned (40 bins) light curve $(400-1000 \mathrm{eV})$ of the XMM-Newton EPIC-pn observation revolution 1086 (November 2005) folded during the timing procedure (described in the text). The solid line represents the combination of three Fourier harmonics chosen for the determination of the phase. The phase shift differs slightly from that from the sine fit (dotted line). The dashed line shows, that a fit with the second harmonic (cosine) is not always sufficient. The error bars denote Poissonian errors.

Our approach is justified since in most of the cases some Fourier coefficients are negligible (see the coefficients from the phase folded light curves of the EPIC-pn observations in Fig. 4 as an example).

The analysis of the Fourier coefficients shows that the pulse profile changes in time, as already reported in Haberl et al. (2006) and de Vries et al. (2004, their Fig. 2), and the evolution continued also after the last observation considered by Haberl et al. $(2006$, MJD $=53700$ days). In order to check that the light curve evolution is independent of the adopted pulse profile template (either a sine as in Haberl et al. 2006, or a truncated Fourier expansion, as in the present work), we performed a principal component analysis (PCA, see e.g. Zane \& Turolla 2006, for more details) of the same 16 EPIC-pn light curves we used before, again binned in 40 phase intervals. Starting from the original variables (the 40 values of the counts at the different phases), the PCA computes a new set of variables (the principal components, pcs) which are a linear combination of the old ones and are ordered in such a way that the first pc accounts for the largest variance of the data, the second the second largest, and so on. We find that the first four pcs are actually responsible for $\sim 92 \%$ of the variance, pulse profiles can be effectively classified in terms of only a limited number of pcs which embody their main characteristics. The time evolution of the first four pcs is shown in Fig. 5. The significant changes in both sets of coefficients (pc and Fourier) is a strong evidence of a genuine variation of the pulse profile in the hard band in time. The second last XMM-Newton observation (rev. 1700) that was contaminated by strong flares deviates from the trends in Figs. 4 and 5. As discussed in Zane \& Turolla (2006), the first pc $\left(Z_{1}\right)$ is related to the pulse amplitude, $Z_{2}$ provide a measure of the phase interval of the light maximum and $Z_{3}$ reflects the lightcurve parity with respect to the half period (see Fig. 4 of Zane \& Turolla 2006, for the dependence on the phase of the first four coefficients $v_{i k}$, with $i=1, \ldots, 4$, used to calculate the PCs from the original variables). Although a detailed analysis will not be attempted here, Fig. 5 shows that the largest changes occur in $Z_{1}$ and $Z_{2}$

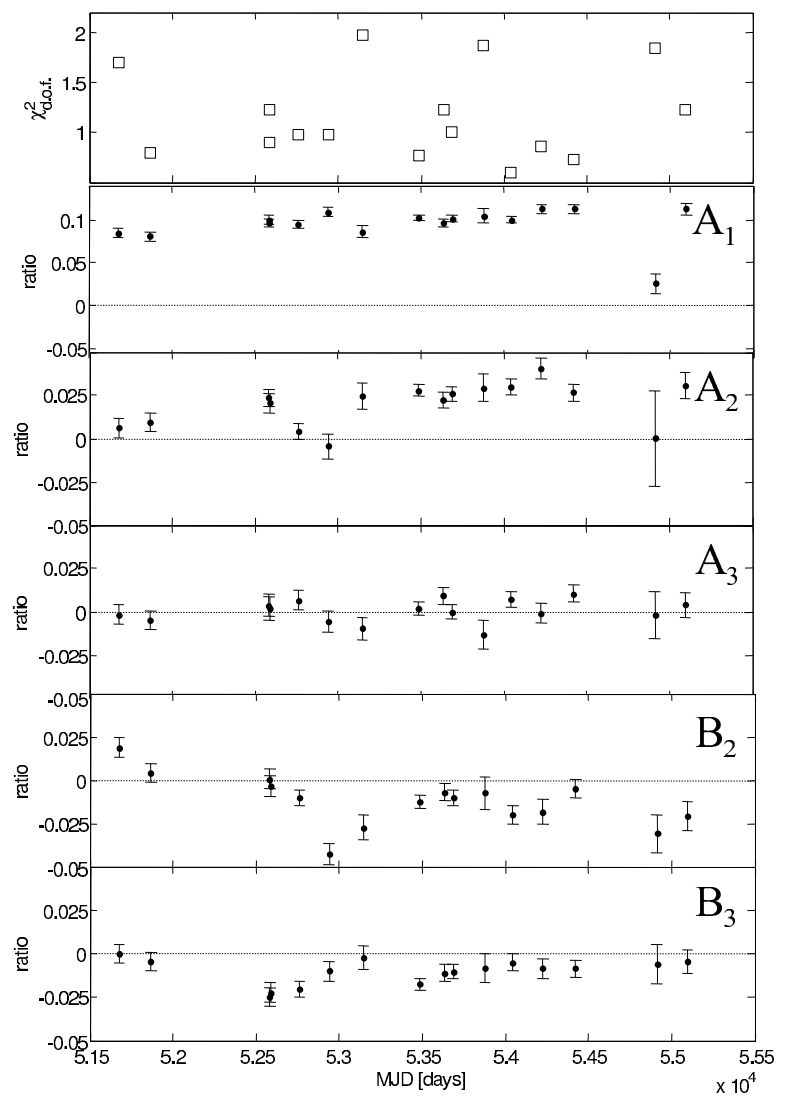

Fig. 4. The Fourier coefficients ( $\cos 1=0$ by definition) of the fit to the phase folded light curves (40 bins) of the EPIC-pn observations. The values of the coefficients are divided by the constant offset $A_{0}$, since the observations have different numbers of photons. The first panel shows the corresponding values of $\chi^{2} /$ d.o.f. All observations have less than $1 \%$ background counts and are filtered using standard GTI files. In case of rev. 1700 ( $\sim 3 \%$ high background) an additional filtering is applied (see Sect. 2) leading to the deviating point near $\mathrm{MJD}=54900$ days. All error bars denote $1 \sigma$ confidence level.

and are then associated to the amplitude and the position of the maximum of the pulses.

\section{Results}

We performed the phase coherent timing by binning the light curves into $10,12,16,18$ and 20 phase bins. A large number of bins leads to large scatter in the Chandra light curves due to the small count rate compared to XMM-Newton while too few bins result in an insufficient time resolution ${ }^{2}$. We derived the best timing solution for all observations (excluding ROSAT data and the HRC observation 7251) for 12 phase bins in order to minimise the phase residuals.

If we fit the phase folded light curves with a pure sinusoid, we obtain $P=8.3911153307(22) \mathrm{s}, \dot{f}=-9.933(52) \times$ $10^{-16} \mathrm{~Hz} / \mathrm{s}$ for the phase coherent timing solution. This corresponds to $\chi^{2} /$ d.o.f. $=12.7$ for the timing solution and for the light curve fits we obtain $\chi^{2} /$ d.o.f. $=2.29$ on average. Fitting the light curves with a variable combination of the three Fourier harmonics, the result does not change significantly. We obtain $P=8.3911153362(39) \mathrm{s}$ and $\dot{f}=-9.946(74) \times 10^{-16} \mathrm{~Hz} / \mathrm{s}$, but

\footnotetext{
${ }^{2}$ Note that the time resolution for the MOS detectors is $0.3 \mathrm{~s}$ (small window), $0.9 \mathrm{~s}$ (large window) and $2.6 \mathrm{~s}$ (full frame), i.e. less than one phase bin. This is compensated by the large number of photons. KvK05 used 16 phase bins (including the MOS detectors).
} 


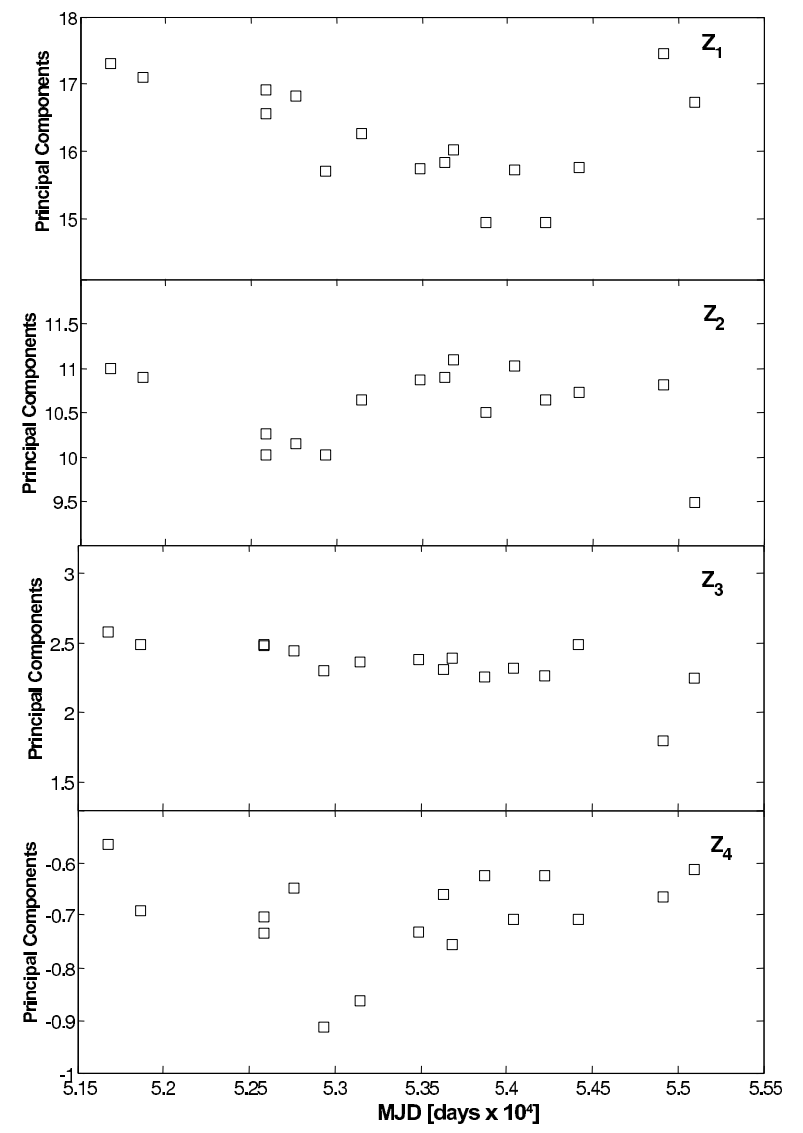

Fig. 5. The principal components (see Zane \& Turolla 2006 for details) of the 16 EPIC-pn observations of RX J0720.4-3125.

the phase residuals have smaller errors and therefore the timing solution corresponds to $\chi^{2} /$ d.o.f. $=47$. For the light curve fits we obtain $\chi^{2} /$ d.o.f. $=1.23$ on average.

Although the periods determined from the ROSAT data (see Zane et al. 2002 and Cropper et al. 2004 for details) differ significantly to the reference period $P_{2}$, we include these data sets, except the All-Sky Survey (where we find no period), for a further timing solution. The inclusion of the ROSAT data extends the time span of observations from $\approx 9.6 \mathrm{yrs}$ to $\approx 16.5 \mathrm{yrs}$ and enlarges the data set from 64 to 70 observations. Again, we fitted the light curves with a variable combination of the three Fourier harmonics and obtain $P=8.3911153336(22) \mathrm{s}$ and $\dot{f}=-9.961(67) \times 10^{-16} \mathrm{~Hz} / \mathrm{s}$ for 12 phase bins. This corresponds to $\chi^{2} /$ d.o.f. $=47$ for the timing solution and for the light curve fits we obtain $\chi^{2} /$ d.o.f. $=1.17$ on average.

For a third timing solution we excluded the Chandra data, but using the ROSAT data (58 data sets out of 70) and the same reference time and period as for the other two timing solutions. Fitting the light curves with a variable combination of the three Fourier harmonics, we obtain $P=8.3911153310(22)$ s and $\dot{f}=$ $-9.940(71) \times 10^{-16} \mathrm{~Hz} / \mathrm{s}$ for 12 phase bins that corresponds to $\chi^{2} /$ d.o.f. $=45$ for the timing solution and for the light curve fits we obtain $\chi^{2} /$ d.o.f. $=1.21$ on average.

To verify the correctness of our timing solutions we apply the $Z_{1}^{2}$ method to the same combination of observations as for the phase coherent timing solutions (see Fig. 6). Using all 70 data sets, we found a maximum at $P=8.391115309(14) \mathrm{s}$ and $\dot{f}=-9.992(61) \times 10^{-16} \mathrm{~Hz} / \mathrm{s}$ with $Z_{1}^{2}=8263$. The errors for $P$ and $\dot{f}$ are obtained from the equation in Ransom et al. (2002), whereas the values correspond to the maximum peak in the center of the $1 \sigma$ confidence region. In this region the peak is symmetric. Finally, the errors are scaled to $\sqrt{\chi^{2} / \text { d.o.f. }}$

The results of the three phase coherent timing solutions and from the $Z_{1}^{2}$ method are summarised in Table 3. Note that our results are only slightly different from previous solutions (but scatter less), i.e. the restriction of our investigations to the hard band reduces the phase residuals and influences the shape of their evolution in time, but does not affect basic parameters like the spin-down age or magnetic field strength of RX J0720.4-3125 significantly. For completeness the results from the coherent "all data" solution and the $Z_{1}^{2}$ method for the soft photons are listed in Table 3 too, illustrating the difference of the timing solutions in the different bands. The corresponding TOAs from the final solutions are listed in Table 2, last two columns. The errors of the TOAs are derived from the formal fit errors of the phase binned light curves by fitting them to the combination of Fourier harmonics as explained in Sect. 4.

\section{Discussion}

We present new updated phase coherent timing solutions for the isolated radio-quiet X-ray pulsar RX J0720.4-3125 including the most recent XMM-Newton and Chandra observations, restricting our analysis to the hard energy band (400-1000 eV), except for Chandra HRC and ROSAT data. The new solutions were obtained with and without the inclusion of the ROSAT and Chandra data and differ slightly from previous ones (see KvK05, and vK07 and Table 3). We improved the phase determination and the modeling of the phase folded light curves by fitting a variable combination of the three Fourier harmonics with the sine as the leading term, instead of a pure sinusoid. We checked the phase coherent timing solutions applying the $Z_{1}^{2}$ method to the observations in the $P / \dot{f}$ plane. All timing solutions correspond to a $\chi^{2} /$ d.o.f. $\approx 50$, i.e. the phase residuals are still large and a timing solution based only on $\dot{f}$ and $P$ provides an inadequate model for the spin behaviour of RX J0720.4-3125.

Due to the restriction to the hard band for most of the data, the phase residuals from EPIC-pn do not deviate systematically to those from EPIC-MOS1 and MOS2 and Chandra unlike those derived without energy restriction (see Fig. 1 in H09 for comparison and Fig. 1 in this work) and most observations are in good agreement to each other. However, the residual of the Chandra observation 7251 is still not consistent (lowest number of counts and bad statistics) to the other phase residuals and the phase residuals from the XMM-Newton EPIC-pn and EPIC-MOS1 $\&$ MOS2 observations 0622 scatter much. The observations of rev 0622 were all performed in different filters: thick, medium and thin, having different transparency for the softer photons (causing deviations in the phase as discussed before) in the hard band.

Including the most recent Chandra and XMM-Newton observations, the ROSAT data become less important for the timing solution. This explains why our three phase coherent timing solutions are almost identical and scatter less than the phase coherent timing solutions in KvK05 and vK07 (without a glitch).

Applying the timing solutions to the observations of RX J0720.4-3125 we still obtain large residuals. Due to the small number of counts, the ROSAT data are ambiguous in determining the correct phase, the periods differ significantly from the periods of the other observations and the ROSAT periodograms are noisy. However, the inclusion of the ROSAT data extends the observed time span from 9.6 to 16 yrs. Therefore, we present the phase residuals from all observations, but derived 


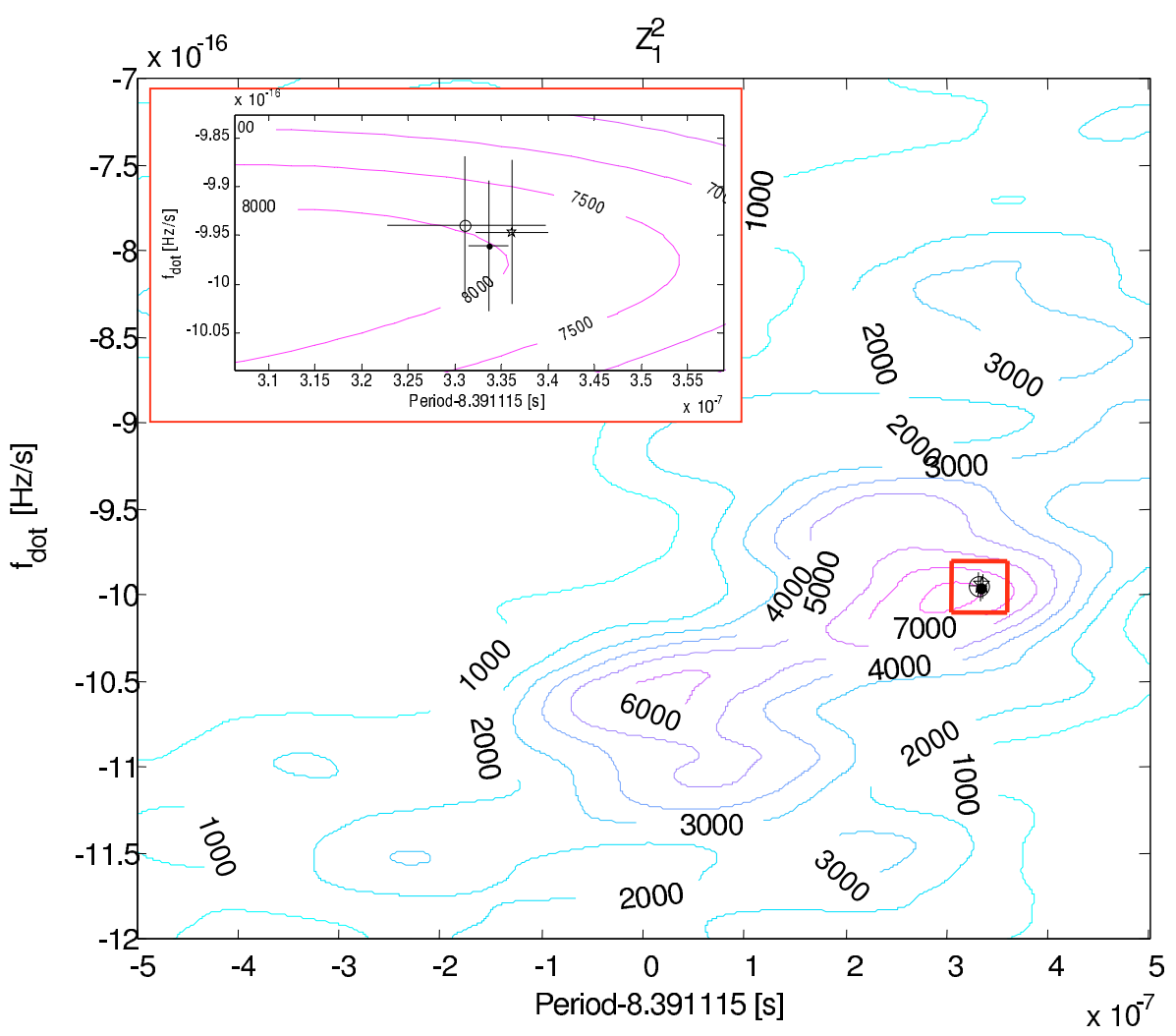

Fig. 6. The $\mathrm{Z}_{1}^{2}$ values in the $P-\dot{f}$ plane derived from the XMM-Newton, ROSAT and Chandra observations (except the HRC observation 7251) of RX J0720.4-3125 compared to the results of the phase coherent timing solutions in this work. The peak is located at $P=8.391115309(14) \mathrm{s}$ and $\dot{f}=-9.992(61) \times$ $10^{-16} \mathrm{~Hz} / \mathrm{s}$. The three different timing solutions derived without the Chandra data (open circle), without ROSAT data (star) and using XMM-Newton, ROSAT and Chandra observations (dot) are not resolved on this scale (red box), but become visible enlarging the red box (shown in the upper left panel); the values are listed in Table 3. The errors of the $Z_{1}^{2}$ solution are scaled to $\sqrt{\chi^{2} / \text { d.o.f. All timing solutions }}$ are derived from the hard band $(400-1000 \mathrm{eV})$.

Table 3. $P$ and $\dot{f}$ derived from different methods in this paper, compared to previous results.

\begin{tabular}{|c|c|c|c|c|c|}
\hline Solution & $\begin{array}{l}\text { Period-8.391115 } \\
\times 10^{-7}[\mathrm{~s}]\end{array}$ & $\begin{array}{l}\dot{f} \\
\times 10^{-16}[\mathrm{~Hz} / \mathrm{s}]\end{array}$ & $\begin{array}{c}\mathrm{rms} \\
{[\mathrm{s}]}\end{array}$ & d.o.f. & $\chi^{2} /$ d.o.f. \\
\hline \multicolumn{6}{|c|}{ This work, hard band } \\
\hline "all data" & $3.336(22)$ & $-9.961(67)$ & 0.62 & $70-3$ & 45 \\
\hline After fitting a sine & & & 0.29 & $70-5$ & 7.8 \\
\hline After fitting an abs(sine) & & & 0.31 & $70-5$ & 8.6 \\
\hline without ROSAT & $3.362(39)$ & $-9.946(74)$ & 0.60 & $64-3$ & 47 \\
\hline After fitting a sine & & & 0.19 & $64-5$ & 5.3 \\
\hline After fitting an abs(sine) & & & 0.21 & $64-5$ & 6.5 \\
\hline without Chandra & $3.310(22)$ & $-9.940(71)$ & 0.61 & $58-3$ & 47 \\
\hline After fitting a sine & & & 0.31 & $58-5$ & 9.7 \\
\hline After fitting an abs(sine) & & & 0.33 & $58-5$ & 8.7 \\
\hline$Z_{1}^{2}$ "all data" & $3.09(14)$ & $-9.992(61)$ & 0.73 & $70-3$ & 64 \\
\hline$Z_{1}^{2}$ without ROSAT & $2.96(13)$ & $-10.047(34)$ & 0.58 & $64-3$ & 46 \\
\hline$Z_{1}^{2}$ without Chandra & $3.09(13)$ & $-9.980(36)$ & 0.72 & $58-3$ & 51 \\
\hline \multicolumn{6}{|c|}{ This work, soft band } \\
\hline "all data" & $3.429(22)$ & $-9.956(72)$ & 0.50 & $70-3$ & 40 \\
\hline$Z_{1}^{2}$ "all data" & $3.31(62)$ & $-9.959(17)$ & 0.50 & $70-3$ & 39 \\
\hline \multicolumn{6}{|c|}{ Previous work (applied to the hard band) } \\
\hline vK07 (“all data") & $2.670(84)$ & $-9.88(13)$ & 0.97 & $70-3$ & 81 \\
\hline vK07 (without ROSAT) & $2.846(77)$ & $-9.74(04)$ & 1.30 & $64-3$ & 207 \\
\hline KvK05 ("all data") & $3.20(13)$ & $-9.918(15)$ & 0.64 & $70-3$ & 56 \\
\hline KvK05 (Chandra) & $3.05(16)$ & $-9.97(06)$ & 0.64 & $12-3$ & 57 \\
\hline
\end{tabular}

Notes. For the timing solution in this work we always excluded the Chandra HRC observation 7251. Since the phase residuals seem to follow a periodic pattern we fitted a sine and an abs(sine), see Sect. 6. All errors correspond to $1 \sigma$ confidence (for the $Z_{1}^{2}$ solution see Ransom et al. 2002

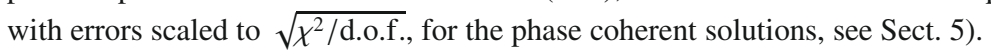

from the timing solution without ROSAT data (hard band) in Fig. 7 shown with an error weighted sine and an abs(sine) fit (see also H09). The phase residuals reached a minimum around MJD = 52800 days and continue towards negative values, maybe a new minimum around MJD = 55300 days; but this has to be confirmed by new observations finding a possible turning point. There is no clear evidence for a minimum in the past (probably MJD = 50000 days including the ROSAT data), but the ROSAT data are less reliable. 


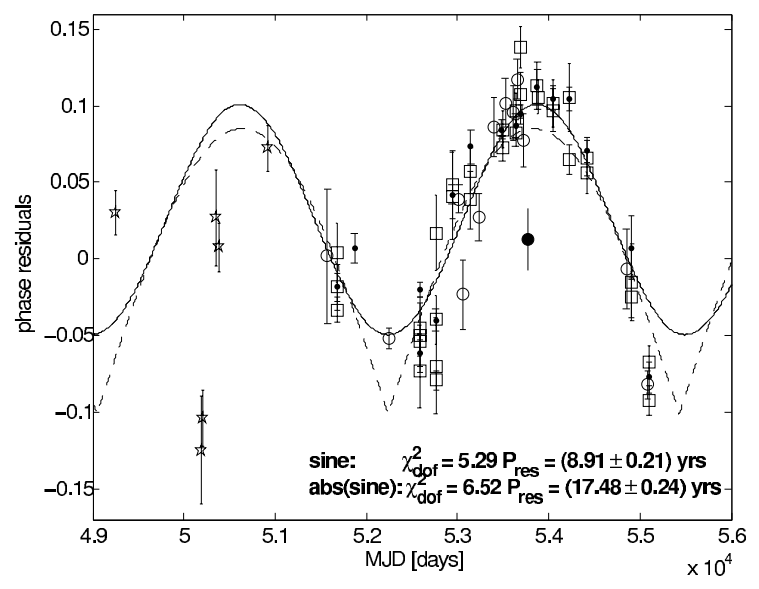

Fig. 7. Phase residuals after applying the timing solutions derived without the ROSAT observations and without the Chandra HRC observation 7251 (marked as a filled circle) from RX J0720.4-3125 applied to all observations listed in Table 1. The best fits with a sine and an abs(sine) model (only the observations used in the current timing solution are used for the fits) are also shown. Squares mark residuals derived from XMM-Newton EPIC-MOS1 and MOS2 observations, dots EPIC-pn observations, circles Chandra HRC and ACIS-CC data and stars ROSAT data. All error bars denote $1 \sigma$.

Fitting a sine or an abs(sine) to the phase residuals reduces the $\chi^{2} /$ d.o.f. from $\approx 50$ to $\approx 6-7$ with a period $P_{\text {res }} \approx 8.9 \mathrm{yrs}^{3}$ (i.e. the observations cover two cycles) or twice this period fitting an abs(sine), respectively. However, the $\chi^{2} /$ d.o.f. is formally still unacceptable. A sinusoidal behaviour of the phase residuals could be explained by precession, as suggested in de Vries et al. (2004) and Haberl et al. (2006). Precession would cause an advanced (the NS precesses towards the observer) and a retarded (the NS precesses backwards with respect to the observer) signal, whereas the residuals would follow a sine (Nelson et al. 1990). During precession the observer would see different parts of the surface that would lead to changing spectral properties, such as changes in temperature and size of the emitting surface. If two, roughly antipodal, hot spots would both contribute to the $\mathrm{X}$-ray emission, the phase residuals caused from both hot spots would be shifted by $P_{\text {res }} / 2$ : if one hot spot precesses towards the observer and appears, the second hot spot moves backwards with respect to the observer and disappears. Since the timing is not sensitive to which of the hot spots is causing the residuals, the phase residuals qualitatively may have the shape of an abs(sine), having twice the period of the corresponding sine (two peaks from two different hot spots). The presence of the larger period is supported by the variable phase lags between hard and soft photons, see Haberl et al. (2004, 2006, and H09). However, it has to be shown whether this scenario can explain the spectral behaviour in $\mathrm{H} 09$ too. Formally, then also the spectral changes undergo a periodic behaviour having $P_{\text {res }} \approx 2 \times 8.9$ yrs. A long term period of $P_{\text {res }} \approx 17-18$ yrs could explain why the spectral changes do not show a periodic trend (H09) yet, since the time span of the XMM-Newton observations would cover less than one cycle. The new turning point of the phase residuals would be at MJD $=55387_{-100}^{+107}$ days (first half of July 2010) or at $\mathrm{MJD}=55240_{-8}^{+8}$ days (first half of February 2010) for the sine or the abs(sine) fit, respectively (see Fig. 7).

\footnotetext{
${ }^{3}$ In H09 long term periods of $P_{\text {res }} \approx 5.5-7.5$ yrs were found for the sine fit. This is significantly less than found in this work. However, the timing solutions used in H09 were not derived from the inclusion of the new data but were adopted from $\mathrm{KvK} 05$ and vK07, also required the inclusion of an additional slope (not necessary in this work).
}

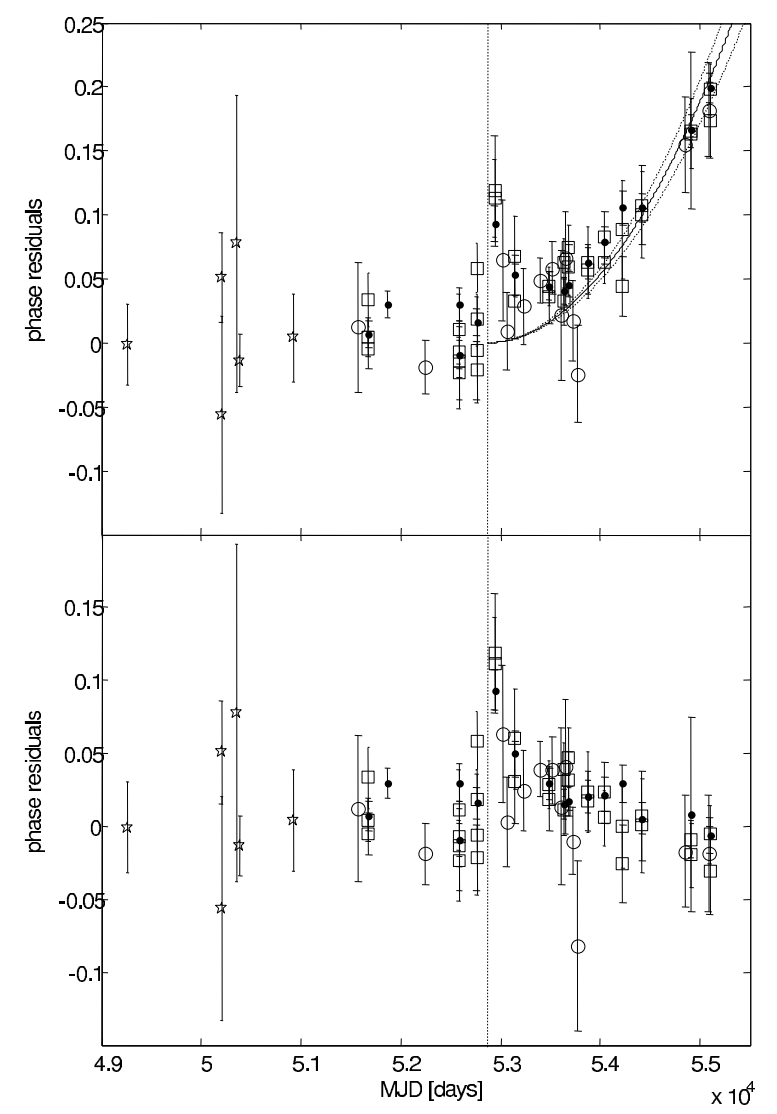

Fig. 8. Upper panel: the phase residuals of RXJ0720.4-3125 after applying the glitch solution ("all data") in vK07. The glitch time $t_{\mathrm{g}}=52866$ days is marked as dotted vertical line. We fitted an error wighted parabolic slope (solid line, dashed lines indicating the $1 \sigma$ confidence range). corresponding to a post-glitch correction of $\dot{f}_{\mathrm{c}}=$ $-1.11(10) \times 10^{-17} \mathrm{~Hz} / \mathrm{s}$. Lower panel: the phase residuals after applying the post-glitch $\dot{f}$ correction. All symbols like in Fig. 7.

Alternatively, vK07 proposed a glitch around MJD = 53000 days explaining the spectral and temporal changes. As shown in H09, the temperature increased at this time, followed by a slow decrease, as expected during and after a glitch event. Therefore vK07 presented a "glitch solution" to explain also the timing behaviour of RXJ0720.4-3125. This glitch solution was not applied correctly in H09. Indeed, the glitch solution presented in vK07 minimises the phase residuals for the observations available at that time (MJD = 53500 days), i.e. the statement (in H09) that it produces large residuals is not correct. However, adding new observations after $\mathrm{MJD}=53500$ days (not available in vK07) and applying the glitch solution correctly, the phase residuals grow to larger values. This can be shown even with the observations available in H09 (MJD = 54421 days) and is even more pronounced using the recent data (until MJD = 55100 days), i.e. the conclusions in $\mathrm{H} 09$ remains the same.

To reduce the residuals, we introduce a new additional parameter with the physical meaning of a post-glitch correction for $\dot{f}$. This can be done by fitting a parabola with respect to the time distance to the proposed glitch time, $t_{\mathrm{g}}=52866$ days, in vK07 ("all data" solution). The error weighted fit for the hard band including all data gives $\dot{f}_{\mathrm{c}}=-1.11(10) \times 10^{-17} \mathrm{~Hz} / \mathrm{s}$ and significantly reduces the phase residuals $\left(\chi^{2} /\right.$ d.o.f. $=2.8$, $\mathrm{rms}=0.31 \mathrm{~s})$. vK07 obtained $\dot{f}_{\mathrm{vK} 07}=-1.04(3) \times 10^{-15} \mathrm{~Hz} / \mathrm{s}$ after $t_{\mathrm{g}}$, the new value would be $\dot{f}_{\text {new }}=\dot{f}_{\mathrm{vK} 07}+\dot{f}_{\mathrm{c}}$, i.e. a modification of the glitch solution in vK07. Due to this correction the glitch hypothesis is still a competing model. 
The phase residuals of RX J0720.4-3125 after applying the glitch solution in vK07 and the post-glitch correction (both for the hard band) are shown in Fig. 8.

Recently, an analysis of timing irregularities of 366 radio pulsars (Hobbs et al. 2010) showed that quasi-periodic structures in the timing residuals are quite usual - at least for radio pulsars. These quasi-periodic structures are dominant for young pulsars (age $\leq 10^{5}$ yrs, note that RX J0720.4-3125 is probably much younger than implied by its characteristic age, see Tetzlaff et al. 2010; Kaplan et al. 2007). In some cases the amplitude of the timing residuals are comparable to those of RX J0720.4-3125 and the timing residuals show "periods" of a few years. These radio pulsars were studied over decades and the data points are much denser in time than in the case of X-ray pulsars, thus the timing residuals of RX J0720.4-3125 may also follow such trends. In such cases (as the authors argue in Hobbs et al. 2010) the timing residuals caused due to "slow glitches" may not be a different phenomenon to that causing the timing irregularities.

The behaviour of RX J0720.4-3125 is still not understood. The monitoring of RX J0720.4-3125 using XMM-Newton and Chandra is still ongoing and will help to bring us closer to understand the reason for its behaviour.

Note added in proof Recently, the object called "Calvera" by Rutledge et al. (2008) was confirmed to be an isolated neutron star based on the measurement of a period of $0.059 \mathrm{~s}$. This indicates that it is most likely a recycled pulsar (Zane et al. 2010).

Acknowledgements. The XMM-Newton project is supported by the Bundesministerium für Wirtschaft und Technologie/Deutsches Zentrum für Luft- und Raumfahrt (BMWI/DLR, FKZ 50 OX 0001) and the Max-Planck Society. M.M.H. acknowledges support by the Deutsche Forschungsgemeinschaft (DFG) through SFB/TR 7 "Gravitationswellenastronomie" and CompStar, a research networking programme of the European Science Foundation (ESF). The work of R.T. is partially funded by INAF-ASI through grant AAE TH-058. We like to thank the referee Marten van Kerkwijk for the fast and detailed review of the manuscript and for useful comments which helped in improving the paper.

\section{References}

Buccheri, R., Bennett, K., Bignami, G. F., et al. 1983, A\&A, 128, 245 Cropper, M., Zane, S., Ramsay, G., Haberl, F., \& Motch, C. 2001, A\&A, 365, L302
Cropper, M., Haberl, F., Zane, S., \& Zavlin, V. E. 2004, MNRAS, 351, 1099 David, L., Harnden, J. F., Kearns, K., \& Zombeck, M. 1996, The ROSAT High Resolution Imager (HRI), USRSDC/SAO Calibration Report, revised de Vries, C. P., Vink, J., Méndez, M., \& Verbunt, F. 2004, A\&A, 415, L31 Eisenbeiss, T., Ginski, C., Hohle, M. M., et al. 2010, Astron. Nachr., 331, 243 Garmire, G. P., Bautz, M. W., Ford, P. G., Nousek, J. A., \& Ricker, Jr., G. R. 2003, in SPIE Conf. Ser. 4851, ed. J. E. Truemper, \& H. D. Tananbaum, 28 Haberl, F. 2007, Ap\&SS, 308, 181

Haberl, F., Motch, C., Buckley, D. A. H., Zickgraf, F. J., \& Pietsch, W. 1997, A\&A, 326, 662

Haberl, F., Zavlin, V. E., Trümper, J., \& Burwitz, V. 2004, A\&A, 419, 1077

Haberl, F., Turolla, R., de Vries, C. P., et al. 2006, A\&A, 451, L17

Hobbs, G., Lyne, A. G., \& Kramer, M. 2010, MNRAS, 402, 1027

Ho, W. C. G., Kaplan, D. L., Chang, P., van Adelsberg, M., \& Potekhin, A. Y. 2007, MNRAS, 375, 821

Hohle, M. M., Haberl, F., Vink, J., et al. 2009, A\&A, 498, 811 (H09)

Kaplan, D. L. 2008, in Astrophysics of Compact Objects, ed. Y.-F. Yuan, X.D. Li, \& D. Lai, Amer. Instit. Phys. Conf. Ser., 968, 129

Kaplan, D. L., \& van Kerkwijk, M. H. 2005, ApJ, 628, L45 (KvK05)

Kaplan, D. L., Kulkarni, S. R., van Kerkwijk, M. H., \& Marshall, H. L. 2002, ApJ, 570, L79

Kaplan, D. L., van Kerkwijk, M. H., Marshall, H. L., et al. 2003, ApJ, 590, 1008

Kaplan, D. L., van Kerkwijk, M. H., \& Anderson, J. 2007, ApJ, 660, 1428

Kovacs, G. 1981, Ap\&SS, 78, 175

Kraft, R. P., Chappell, J. H., Kenter, A. T., et al. 1997, in SPIE Conf. Ser. 3114, ed. O. H. Siegmund, \& M. A. Gummin, 53

Kulkarni, S. R., \& van Kerkwijk, M. H. 1998, ApJ, 507, L49

Medin, Z., \& Lai, D. 2006a, Phys. Rev. A, 74, 062507

Medin, Z., \& Lai, D. 2006b, Phys. Rev. A, 74, 062508

Medin, Z., \& Lai, D. 2007, MNRAS, 382, 1833

Motch, C., \& Haberl, F. 1998, A\&A, 333, L59

Nelson, R. W., Finn, L. S., \& Wasserman, I. 1990, ApJ, 348, 226

Pires, A. M., Motch, C., Turolla, R., Treves, A., \& Popov, S. B. 2009, A\&A, 498, 233

Ransom, S. M., Eikenberry, S. S., \& Middleditch, J. 2002, AJ, 124, 1788

Rutledge, R. E., Fox, D. B., \& Shevchuk, A. H. 2008, ApJ, 672, 1137

Strüder, L., Briel, U., Dennerl, K., et al. 2001, A\&A, 365, L18

Tetzlaff, N., Neuhäuser, R., Hohle, M. M., \& Maciejewski, G. 2010, MNRAS, 402, 2369

Turner, M. J. L., Abbey, A., Arnaud, M., et al. 2001, A\&A, 365, L27

Turolla, R., Zane, S., \& Drake, J. J. 2004, ApJ, 603, 265

van Kerkwijk, M. H., Kaplan, D. L., Pavlov, G. G., \& Mori, K. 2007, ApJ, 659, L149 (vK07)

Walter, F. M., Wolk, S. J., \& Neuhäuser, R. 1996, Nature, 379, 233

Zane, S., \& Turolla, R. 2006, MNRAS, 366, 727

Zane, S., Haberl, F., Cropper, M., et al. 2002, MNRAS, 334, 345

Zane, S., Turolla, R., \& Drake, J. J. 2004, Adv. Space Res., 33, 531

Zane, S., Turolla, R., \& Page, D. 2007, Isolated Neutron Stars: from the Surface to the Interior

Zane, S., Mignani, R. P., Turolla, R., et al. 2008, ApJ, 682, 487

Zane, S., Haberl, F., Israel, G. L., et al. 2010, MNRAS, in press, [arXiv: 1009.0209] 
A\&A 521, A11 (2010)

Table 1. All observations of RX J0720.4-3125 listed in chronological order.

\begin{tabular}{|c|c|c|c|c|c|c|}
\hline Obs. Id. & Instrument/setup & $\begin{array}{c}\text { Counts } \\
(120-400 \mathrm{eV})\end{array}$ & $\begin{array}{c}\text { Counts } \\
(400-1000 \mathrm{eV})\end{array}$ & [days] & Start date & $\begin{array}{r}\text { Effective } \\
\text { exposure } \\
{[\mathrm{ks}]}\end{array}$ \\
\hline rp300338n00 & PSPC & & 374 & 49258 & 1993 Sep. 27 & 3.22 \\
\hline rh300508n00 & HRI & & 259 & 50199 & 1996 Apr. 25 & 3.13 \\
\hline rh180100n00 & HRI & & 197 & 50211 & 1996 May 7 & 3.57 \\
\hline rh300508n01 & HRI & & 93 & 50354 & 1996 Sep. 27 & 1.41 \\
\hline rh400884n00 & HRI & & 381 & 50391 & 1996 Nov. 3 & 33.57 \\
\hline h400944n00 & HRI & & 54 & 50924 & 1998 Apr. 20 & 3.57 \\
\hline $368^{1}$ & HRC-S/LETG & & 722 & 51575 & 2000 Feb. 1 & 5.40 \\
\hline $745^{1}$ & HRC-S/LETG & & 392 & 51576 & 2000 Feb. 2 & 26.26 \\
\hline $369^{1}$ & HRC-S/LETG & & 660 & 51579 & 2000 Feb. 4 & 6.12 \\
\hline $0078 \mathrm{~S} 3$ & EPIC-pn/FF thin & 241783 & 115287 & 51677 & 2000 May 13 & 58.60 \\
\hline $\mathrm{S} 1$ & EPIC-MOS1/FF thin & 69623 & 43977 & 51677 & 2000 May 13 & 61.98 \\
\hline $\mathrm{S} 2$ & EPIC-MOS2/SW thin & 68010 & 40818 & 51677 & 2000 May 13 & 61.99 \\
\hline 0175 S3 & EPIC-pn/FF med & 103491 & 55743 & 51870 & 2000 Nov. 21 & 25.65 \\
\hline S7 & EPIC-MOS1/LW med & 15765 & 11662 & 51870 & 2000 Nov. 21 & 18.00 \\
\hline $2774^{2}$ & ACIS-CC & 13455 & 15648 & 52248 & 2001 Dec. 4 & 15.01 \\
\hline $2773^{2}$ & ACIS-CC & 9452 & 11646 & 52248 & 2001 Dec. 5 & 10.61 \\
\hline $2771^{2}$ & ACIS-CC & 876 & 1553 & 52250 & 2001 Dec. 6 & 1.86 \\
\hline $2772^{2}$ & ACIS-CC & 3537 & 4487 & 52250 & 2001 Dec. 6 & 4.05 \\
\hline $0533 \mathrm{~S} 3$ & EPIC-pn/FF thin & 137951 & 72150 & 52585 & 2002 Nov. 6 & 28.38 \\
\hline $\mathrm{S} 1$ & EPIC-MOS1/FF thin & 27987 & 21892 & 52585 & 2002 Nov. 6 & 29.99 \\
\hline $\mathrm{S} 2$ & EPIC-MOS2/FF thin & 29169 & 22192 & 52585 & 2002 Nov. 6 & 29.99 \\
\hline 0534 S3 & EPIC-pn/FF thin & 145057 & 76095 & 52587 & 2002 Nov. 8 & 30.18 \\
\hline $\mathrm{S} 1$ & EPIC-MOS1/FF thin & 28168 & 22112 & 52587 & 2002 Nov. 8 & 31.80 \\
\hline $\mathrm{S} 2$ & EPIC-MOS2/FF thin & 28954 & 22768 & 52587 & 2002 Nov. 8 & 31.79 \\
\hline $0622 \mathrm{U} 2$ & EPIC-pn/SW thick & 123199 & 94655 & 52762 & 2003 May 2 & 72.79 \\
\hline S5 & EPIC-MOS1/FF med & 21204 & 18741 & 52762 & 2003 May 2 & 29.98 \\
\hline S3 & EPIC-MOS1/FF thin & 28433 & 23754 & 52762 & 2003 May 2 & 33.59 \\
\hline S6 & EPIC-MOS2/FF med & 21988 & 19650 & 52762 & 2003 May 2 & 29.99 \\
\hline $\mathrm{S} 4$ & EPIC-MOS2/FF thin & 30901 & 24413 & 52762 & 2003 May 2 & 33.59 \\
\hline $0711 \mathrm{~S} 8$ & EPIC-pn/SW med & 83151 & 60557 & 52940 & 2003 Oct 27 & 24.90 \\
\hline S3 & EPIC-MOS1/FF thin & 10841 & 11937 & 52940 & 2003 Oct. 27 & 13.90 \\
\hline S5 & EPIC-MOS1/LW thin & 10547 & 11769 & 52940 & 2003 Oct. 27 & 15.71 \\
\hline S4 & EPIC-MOS2/FF thin & 11649 & 12392 & 52940 & 2003 Oct. 27 & 13.91 \\
\hline S6 & EPIC-MOS2/LW thin & 11943 & 12382 & 52940 & 2003 Oct. 27 & 15.71 \\
\hline $4666^{3}$ & ACIS-CC & 5606 & 13094 & 53010 & 2004 Jan. 6 & 10.12 \\
\hline $4667^{3}$ & ACIS-CC & 2678 & 5835 & 53011 & 2004 Jan. 7 & 4.79 \\
\hline $4668^{3}$ & ACIS-CC & 2121 & 4918 & 53016 & 2004 Jan. 11 & 5.16 \\
\hline $4669^{3}$ & ACIS-CC & 1890 & 4277 & 53023 & 2004 Jan. 19 & 5.22 \\
\hline $5305^{4}$ & HRC-S/LETG & \multicolumn{2}{|c|}{13275} & 53062 & 2004 Feb. 27 & 35.70 \\
\hline $0815 \mathrm{~S} 1$ & EPIC-pn/FF thin & 130683 & 93280 & 53147 & 2004 May 22 & 41.30 \\
\hline $\mathrm{S} 2$ & EPIC-MOS1/FF thin & 35853 & 41178 & 53147 & 2004 May 22 & 45.21 \\
\hline S3 & EPIC-MOS2/FF thin & 35915 & 42318 & 53147 & 2004 May 22 & 45.22 \\
\hline $4670^{5}$ & ACIS-CC & 5723 & 12329 & 53221 & 2004 Aug. 3 & 10.13 \\
\hline $4671^{5}$ & ACIS-CC & 2778 & 6318 & 53223 & 2004 Aug. 5 & 5.15 \\
\hline $4672^{5}$ & ACIS-CC & 2483 & 5789 & 53227 & 2004 Aug. 9 & 5.12 \\
\hline $4673^{5}$ & ACIS-CC & 2527 & 6034 & 53244 & 2004 Aug. 23 & 5.13 \\
\hline $5581^{6}$ & HRC-S/LETG & \multicolumn{2}{|c|}{30998} & 53393 & 2005 Jan. 23 & 68.20 \\
\hline $0986 \mathrm{~S} 3$ & EPIC-pn/FF thin & 181754 & 131146 & 53488 & 2005 Apr. 28 & 51.43 \\
\hline $\mathrm{S} 1$ & EPIC-MOS1/SW thin & 32400 & 36475 & 53488 & 2005 Apr. 28 & 53.05 \\
\hline S2 & EPIC-MOS2/SW thin & 34456 & 38266 & 53488 & 2005 Apr. 28 & 53.06 \\
\hline $5582^{7}$ & HRC-S/LETG & \multicolumn{2}{|c|}{35777} & 53523 & 2005 Jun. 1 & 70.17 \\
\hline $6364^{8}$ & HRC-S/LETG & \multicolumn{2}{|c|}{22210} & 53610 & 2005 Aug. 27 & 38.87 \\
\hline $1060 \mathrm{~S} 3$ & EPIC-pn/FF thin & 175481 & 120913 & 53636 & 2005 Sep. 23 & 51.14 \\
\hline $\mathrm{S} 1$ & EPIC-MOS $1 / S W$ thin & 33710 & 37070 & 53636 & 2005 Sep. 23 & 52.76 \\
\hline S2 & EPIC-MOS2/SW thin & 33977 & 37706 & 53636 & 2005 Sep. 23 & 52.77 \\
\hline $6369^{9}$ & HRC-S/LETG & \multicolumn{2}{|c|}{7696} & 53652 & 2005 Oct. 8 & 26.26 \\
\hline $7177^{9}$ & HRC-S/LETG & \multicolumn{2}{|c|}{2532} & 53653 & 2005 Oct. 9 & 8.04 \\
\hline $1086 \mathrm{~S} 3$ & EPIC-pn/FF thin & 167725 & 117828 & 53687 & 2005 Nov. 12 & 37.84 \\
\hline $\mathrm{S} 1$ & EPIC-MOS1/SW thin & 25265 & 27091 & 53687 & 2005 Nov. 12 & 39.46 \\
\hline S2 & EPIC-MOS2/SW thin & 25045 & 27580 & 53687 & 2005 Nov. 12 & 39.47 \\
\hline $7243^{10}$ & HRC-S/LETG & \multicolumn{2}{|c|}{5931} & 53718 & 2005 Dec. 14 & 17.18 \\
\hline $7244^{10}$ & HRC-S/LETG & \multicolumn{2}{|c|}{4927} & 53718 & 2005 Dec. 15 & 16.29 \\
\hline $7245^{10}$ & HRC-S/LETG & \multicolumn{2}{|c|}{5249} & 53718 & 2005 Dec. 16 & 17.19 \\
\hline $5584^{10}$ & HRC-S/LETG & \multicolumn{2}{|c|}{5321} & 53718 & 2005 Dec. 17 & 14.19 \\
\hline
\end{tabular}


Table 1. continued.

\begin{tabular}{|c|c|c|c|c|c|c|}
\hline Obs. Id. & Instrument/setup & $\begin{array}{c}\text { Counts } \\
(120-400 \mathrm{eV})\end{array}$ & $\begin{array}{c}\text { Counts } \\
(400-1000 \mathrm{eV})\end{array}$ & $\begin{array}{l}\text { MJD } \\
\text { [days] }\end{array}$ & Start date & $\begin{array}{r}\text { Effective } \\
\text { exposure } \\
{[\mathrm{ks}]}\end{array}$ \\
\hline 7251 & HRC-S/LETG & \multicolumn{2}{|c|}{4787} & 53775 & 2006 Sep. 9 & 10.65 \\
\hline $1181 \mathrm{~S} 1$ & EPIC-pn/FF thin & 89015 & 59524 & 53877 & 2006 May 22 & 20.04 \\
\hline $\mathrm{S} 2$ & EPIC-MOS1/SW thin & 13652 & 14219 & 53877 & 2006 May 22 & 21.66 \\
\hline S3 & EPIC-MOS2/SW thin & 15045 & 15048 & 53877 & 2006 May 22 & 21.66 \\
\hline $1265 \mathrm{~S} 1$ & EPIC-pn/FF thin & 89852 & 60883 & 54044 & 2006 Nov. 5 & 20.04 \\
\hline $\mathrm{S} 2$ & EPIC-MOS1/FF thin & 12414 & 12296 & 54044 & 2006 Nov. 5 & 21.61 \\
\hline S3 & EPIC-MOS2/FF thin & 15291 & 14653 & 54044 & 2006 Nov. 5 & 21.62 \\
\hline $1356 \mathrm{~S} 1$ & EPIC-pn/FF thin & 89411 & 57483 & 54226 & 2007 May 5 & 20.04 \\
\hline $\mathrm{S} 2$ & EPIC-MOS1/FF thin & 16690 & 16685 & 54226 & 2007 May 5 & 21.61 \\
\hline S3 & EPIC-MOS2/FF thin & 17147 & 16818 & 54226 & 2007 May 5 & 21.62 \\
\hline $1454 \mathrm{~S} 1$ & EPIC-pn/FF thin & 102917 & 64833 & 54421 & 2007 Nov. 17 & 23.06 \\
\hline $\mathrm{S} 2$ & EPIC-MOS1/FF thin & 18674 & 18197 & 54421 & 2007 Nov. 17 & 24.62 \\
\hline S3 & EPIC-MOS2/FF thin & 19029 & 18416 & 54421 & 2007 Nov. 17 & 24.62 \\
\hline $10861^{11}$ & HRC-S/LETG & \multirow{2}{*}{\multicolumn{2}{|c|}{$\begin{array}{c}4240 \\
14053\end{array}$}} & 54851 & 2009 Jan 20 & 11.91 \\
\hline $10700^{11}$ & HRC-S/LETG & & & 54876 & 2009 Feb. 14 & 21.82 \\
\hline $1700 \mathrm{~S} 3$ & EPIC-pn/FF thin & 37085 & 30806 & 54913 & 2009 Mar. 21 & 10.84 \\
\hline U2 & EPIC-MOS1/FF thin & 11481 & 11876 & 54912 & 2009 Mar. 21 & 17.69 \\
\hline $\mathrm{U} 2$ & EPIC-MOS2/FF thin & 11936 & 11556 & 54912 & 2009 Mar. 21 & 17.69 \\
\hline $10701^{12}$ & HRC-S/LETG & \multicolumn{2}{|c|}{15647} & 55086 & 2009 Sep. 11 & 33.17 \\
\hline 1792 S3 & EPIC-pn/FF thin & 65407 & 48105 & 55096 & 2009 Sep. 22 & 17.90 \\
\hline $\mathrm{S} 1$ & EPIC-MOS1/SW thin & 12524 & 11919 & 55096 & 2009 Sep. 22 & 19.22 \\
\hline S2 & EPIC-MOS2/SW thin & 12724 & 11993 & 55096 & 2009 Sep. 22 & 19.24 \\
\hline
\end{tabular}

Notes. Observation 7251, in italic, is not used in this work (see Sect. 3). The identifier marks the merged data sets: observations with the same tag are merged into one data set. Counts for ROSAT and Chandra HRC were not divided into soft (120-400 eV) and hard (400-1000 eV) band (see Sect. 3), hence we list the total number of counts. The count numbers for the HRC observations are comparable to those listed in KvK05, but are a factor 100 less than those listed in vK07 (probably due to missprint in vK07) although the data handling in all three works (KvK05, vK07 and this work) is comparable. The count numbers of all other observations are in good agreement. For details on ROSAT and XMM-Newton observations we refer to Cropper et al. (2004) and (Hohle et al. 2009 and Sects. 2, 3 this work), respectively. 
A\&A 521, A11 (2010)

Table 2. Individual periods and TOAs of the observations of RX J0720.4-3125 as listed in Table 1.

\begin{tabular}{|c|c|c|c|c|c|}
\hline Obs. Id. & Instrument/setup & $\begin{array}{l}\text { Period } \\
(120-400 \mathrm{eV}) \\
{[\mathrm{s}]}\end{array}$ & $\begin{array}{l}\text { Period } \\
(400-1000 \mathrm{eV}) \\
{[\mathrm{s}]}\end{array}$ & $\begin{array}{l}\text { TOA } \\
(120-400 \mathrm{eV}) \\
\text { [days] }\end{array}$ & $\begin{array}{l}\text { TOA } \\
(400-1000 \mathrm{eV}) \\
\text { [days] }\end{array}$ \\
\hline rp300338n00 & PSPC & \multicolumn{2}{|c|}{$8.39120(44)$} & $49257.2547153(16)$ & $49257.2547298(21)$ \\
\hline rh300508n00 & HRI & \multicolumn{2}{|c|}{$8.3852(63)$} & $50198.6873383(25)$ & $50198.6873509(35)$ \\
\hline rh180100n00 & HRI & \multicolumn{2}{|c|}{$8.3443(11)$} & $50210.5562791(18)$ & $50210.5562812(17)$ \\
\hline rh300508n01 & HRI & \multicolumn{2}{|c|}{$8.4902(98)$} & $50353.9975633(27)$ & $50353.9975702(20)$ \\
\hline rh400884n00 & HRI & \multicolumn{2}{|c|}{$8.391130(50)$} & $50391.3004644(14)$ & $50391.3004729(18)$ \\
\hline h400944n00 & HRI & \multicolumn{2}{|c|}{$8.3921(10)$} & $50925.6878393(11)$ & $50925.6878472(21)$ \\
\hline $368^{1}$ & HRC-S/LETG & \multicolumn{2}{|c|}{$8.39063(49)$} & \multirow[t]{3}{*}{$51577.0395641(21)$} & \multirow{3}{*}{$51577.0395693(16)$} \\
\hline $745^{1}$ & HRC-S/LETG & \multirow[b]{3}{*}{$8.391113(18)$} & \multirow[b]{3}{*}{$8.391085(35)$} & & \\
\hline $369^{1}$ & HRC-S/LETG & & & & \\
\hline $0078 \mathrm{~S} 3$ & EPIC-pn/FF thin & & & $51677.44324000(30)$ & $51677.44323979(69)$ \\
\hline $\mathrm{S} 1$ & EPIC-MOS1/FF thin & $8.391090(45)$ & $8.391090(55)$ & $51677.47179274(83)$ & $51677.47179286(78)$ \\
\hline $\mathrm{S} 2$ & EPIC-MOS2/SW thin & $8.391113(23)$ & $8.391033(53)$ & $51677.47179234(54)$ & $51677.4717923(13)$ \\
\hline $0175 \mathrm{~S} 3$ & EPIC-pn/FF med & $8.391268(58)$ & $8.39124(12)$ & $51869.95710586(60)$ & $51869.95710803(84)$ \\
\hline S7 & EPIC-MOS1/LW med & $8.39122(22)$ & $8.39107(32)$ & $51869.9949836(13)$ & $51869.9949874(27)$ \\
\hline $2774^{2}$ & ACIS-CC & \multirow{4}{*}{$8.391093(13)$} & \multirow{4}{*}{$8.391133(13)$} & \multirow{4}{*}{$52248.6767290(15)$} & $52248.67672069(69)$ \\
\hline $2773^{2}$ & ACIS-CC & & & & \\
\hline $2771^{2}$ & ACIS-CC & & & & \\
\hline $2772^{2}$ & ACIS-CC & & & & \\
\hline $0533 \mathrm{~S} 3$ & EPIC-pn/FF thin & $8.391143(43)$ & $8.391098(73)$ & $52584.92605120(69)$ & $52584.9260530(10)$ \\
\hline $\mathrm{S} 1$ & EPIC-MOS1/FF thin & $8.39136(12)$ & $8.39088(16)$ & $52584.91993465(11)$ & $52584.9200294(15)$ \\
\hline $\mathrm{S} 2$ & EPIC-MOS2/FF thin & $8.39122(12)$ & $8.39104(20)$ & $52584.91993465(95)$ & $52584.9199325(16)$ \\
\hline $0534 \mathrm{~S} 3$ & EPIC-pn/FF thin & $8.391225(40)$ & $8.391317(63)$ & $52587.00129529(39)$ & $52587.00129506(97)$ \\
\hline $\mathrm{S} 1$ & EPIC-MOS1/FF thin & $8.39119(11)$ & $8.39116(18)$ & $52586.9952779(18)$ & $52586.9952766(29)$ \\
\hline $\mathrm{S} 2$ & EPIC-MOS2/FF thin & $8.39137(13)$ & $8.39137(23)$ & $52586.99527264(93)$ & $52586.9952735(14)$ \\
\hline $0622 \mathrm{U} 2$ & EPIC-pn/SW thick & $8.391133(18)$ & $8.391138(23)$ & $52761.99514720(82)$ & $52761.99514812(64)$ \\
\hline S5 & EPIC-MOS1/FF med & $8.39130(16)$ & $8.39100(20)$ & $52762.2413478(13)$ & $52762.2413532(20)$ \\
\hline S3 & EPIC-MOS1/FF thin & $8.39101(13)$ & $8.39100(17)$ & $52761.8668566(19)$ & $52761.8668516(28)$ \\
\hline S6 & EPIC-MOS2/FF med & $8.39100(15)$ & $8.39126(15)$ & $52762.2413439(17)$ & $52762.24144068(14)$ \\
\hline $\mathrm{S} 4$ & EPIC-MOS2/FF thin & $8.390950(95)$ & $8.39118(21)$ & $52761.8669520(11)$ & $52761.8669491(20)$ \\
\hline $0711 \mathrm{~S} 8$ & EPIC-pn/SW med & $8.391082(48)$ & $8.391188(82)$ & $52940.11635982(97)$ & $52940.11627407(69)$ \\
\hline S3 & EPIC-MOS1/FF thin & $8.39125(31)$ & $8.39077(52)$ & $52939.99012307(84)$ & $52939.9900211(20)$ \\
\hline S5 & EPIC-MOS1/LW thin & $8.39087(24)$ & $8.39059(41)$ & $52940.1679353(12)$ & $52940.1679433(29)$ \\
\hline S4 & EPIC-MOS2/FF thin & $8.39169(32)$ & $8.39051(49)$ & $52939.9900070(17)$ & $52939.9900222(24)$ \\
\hline S6 & EPIC-MOS2/LW thin & $8.39091(22)$ & $8.39147(26)$ & $52940.1679318(13)$ & $52940.1679407(14)$ \\
\hline $4666^{3}$ & ACIS-CC & $8.3911142(23)$ & $8.3911216(28)$ & $53016.68131016(91)$ & $53,016.6813159(12)$ \\
\hline $4667^{3}$ & ACIS-CC & & & & \\
\hline $4668^{3}$ & ACIS-CC & & & & \\
\hline $4669^{3}$ & ACIS-CC & & & & \\
\hline $5305^{4}$ & HRC-S/LETG & 8.39 & $9(25)$ & $53062.4157092(15)$ & $53062.4157091(15)$ \\
\hline $0815 \mathrm{~S} 1$ & EPIC-pn/FF thin & $8.391105(35)$ & $8.391143(58)$ & $53147.68119387(43)$ & $53147.6812041(15)$ \\
\hline $\mathrm{S} 2$ & EPIC-MOS1/FF thin & $8.391108(68)$ & $8.39111(15)$ & $53147.6883801(11)$ & $53147.6883883(31)$ \\
\hline S3 & EPIC-MOS2/FF thin & $8.391150(60)$ & $8.39120(13)$ & $53147.68838022(93)$ & $53147.6883911(21)$ \\
\hline $4670^{5}$ & ACIS-CC & $8.3911146(15)$ & $8.3911165(16)$ & $53,230.5756209(11)$ & $53230.5756243(19)$ \\
\hline $4671^{5}$ & ACIS-CC & & & & \\
\hline $4672^{5}$ & ACIS-CC & & & & \\
\hline $4673^{5}$ & ACIS-CC & & & & \\
\hline $5581^{6}$ & HRC-S/LETG & 8.391 & $10(95)$ & $53,393.6674769(19)$ & $53393.6674775(23)$ \\
\hline $0986 \mathrm{~S} 3$ & EPIC-pn/FF thin & $8.391138(23)$ & $8.391140(30)$ & $53488.67561302(44)$ & $53488.67561797(84)$ \\
\hline $\mathrm{S} 1$ & EPIC-MOS $1 / \mathrm{SW}$ thin & $8.391095(45)$ & $8.391008(48)$ & $53488.6695937(14)$ & $53488.6695969(13)$ \\
\hline S2 & EPIC-MOS2/SW thin & $8.391183(38)$ & $8.391018(53)$ & $53488.6696892(13)$ & $53488.6696931(14)$ \\
\hline $5582^{7}$ & HRC-S/LETG & 8.391 & $38(63)$ & $53522.9398395(35)$ & $53522.9398385(29)$ \\
\hline $6364^{8}$ & HRC-S/LETG & 8.39 & $9(25)$ & $53610.0881154(30)$ & $53610.0881131(20)$ \\
\hline $1060 \mathrm{~S} 3$ & EPIC-pn/FF thin & $8.391120(20)$ & $8.391082(33)$ & $53636.30015746(23)$ & $53636.3001616(10)$ \\
\hline $\mathrm{S} 1$ & EPIC-MOS1/SW thin & $8.391095(40)$ & $8.391130(50)$ & $53636.29413571(70)$ & $53636.2941416(13)$ \\
\hline $\mathrm{S} 2$ & EPIC-MOS2/SW thin & $8.391103(38)$ & $8.391163(58)$ & $53636.29413754(96)$ & $53636.29413962(83)$ \\
\hline $6369^{9}$ & HRC-S/LETG & 8.39 & $0(11)$ & $53652.2601916(17)$ & $53652.2601885(15)$ \\
\hline $7177^{9}$ & HRC-S/LETG & & & & \\
\hline 1086 S3 & EPIC-pn/FF thin & $8.391125(25)$ & $8.391072(38)$ & $53687.17180535(53)$ & $53687.17180817(92)$ \\
\hline $\mathrm{S} 1$ & EPIC-MOS1/SW thin & $8.391003(63)$ & $8.391085(80)$ & $53687.1657835(12)$ & $53687.16588680(52)$ \\
\hline S2 & EPIC-MOS2/SW thin & $8.391200(55)$ & $8.390998(78)$ & $53687.1658825(10)$ & $53687.1658857(12)$ \\
\hline $7243^{10}$ & HRC-S/LETG & 8.391 & $15(10)$ & $53720.0243035(13)$ & $53720.0243020(12)$ \\
\hline $7244^{10}$ & HRC-S/LETG & & & & \\
\hline $7245^{10}$ & HRC-S/LETG & & & & \\
\hline $5584^{10}$ & HRC-S/LETG & & & & \\
\hline
\end{tabular}


Table 2. continued.

\begin{tabular}{|c|c|c|c|c|c|}
\hline Obs. Id. & Instrument/setup & $\begin{array}{l}\text { Period } \\
(120-400 \mathrm{eV}) \\
{[\mathrm{s}]}\end{array}$ & $\begin{array}{l}\text { Period ] } \\
(400-1000 \mathrm{eV}) \\
{[\mathrm{s}]}\end{array}$ & $\begin{array}{l}\text { TOA } \\
(120-400 \mathrm{eV}) \\
\text { [days] }\end{array}$ & $\begin{array}{l}\text { TOA } \\
(400-1000 \mathrm{eV}) \\
\text { [days] }\end{array}$ \\
\hline 7251 & $H R C-S / L E T G$ & \multicolumn{2}{|c|}{$8.39212(88)$} & $53775.3509131(17)$ & $53775.35091144(12)$ \\
\hline $1181 \mathrm{~S} 1$ & EPIC-pn/FF thin & $8.391090(70)$ & $8.39120(11)$ & $53877.32857154(71)$ & $53877.3285752(11)$ \\
\hline $\mathrm{S} 2$ & EPIC-MOS1/SW thin & $8.39137(17)$ & $8.39101(20)$ & $53877.3225489(15)$ & $53877.32255272(65)$ \\
\hline S3 & EPIC-MOS2/SW thin & $8.39131(13)$ & $8.39116(18)$ & $53877.3225491(11)$ & $53877.3225524(16)$ \\
\hline $1265 \mathrm{~S} 1$ & EPIC-pn/FF thin & $8.391075(65)$ & $8.39104(11)$ & $54044.60537610(44)$ & $54044.6053791(11)$ \\
\hline $\mathrm{S} 2$ & EPIC-MOS1/FF thin & $8.39117(16)$ & $8.39074(17)$ & $54044.5990605(12)$ & $54044.5990664(13)$ \\
\hline S3 & EPIC-MOS2/FF thin & $8.39099(13)$ & $8.39096(19)$ & $54044.59906220(62)$ & $54044.5990665(18)$ \\
\hline $1356 \mathrm{~S} 1$ & EPIC-pn/FF thin & $8.391150(65)$ & $8.391113(98)$ & $54225.84098865(82)$ & $54225.84099190(98)$ \\
\hline S2 & EPIC-MOS1/FF thin & $8.39107(21)$ & $8.39066(27)$ & $54225.8345794(18)$ & $54225.83457862(99)$ \\
\hline S3 & EPIC-MOS2/FF thin & $8.39103(23)$ & $8.39096(22)$ & $54225.8346806(13)$ & $54225.8346782(22)$ \\
\hline 1454 S1 & EPIC-pn/FF thin & $8.391130(50)$ & $8.391085(85)$ & $54421.36989356(53)$ & $54421.36989479(60)$ \\
\hline S2 & EPIC-MOS1/FF thin & $8.39090(16)$ & $8.39109(17)$ & $54421.3635797(17)$ & $54421.3635840(15)$ \\
\hline S3 & EPIC-MOS2/FF thin & $8.39131(22)$ & $8.39127(22)$ & $54421.3635875(20)$ & $54421.3635849(18)$ \\
\hline $10861^{11}$ & HRC-S/LETG & \multicolumn{2}{|c|}{$8.39129(20)$} & $54863.7916775(17)$ & $54863.7916751(12)$ \\
\hline $10700^{11}$ & HRC-S/LETG & & & & \\
\hline $1700 \mathrm{~S} 3$ & EPIC-pn/FF thin & $8.39108(99)$ & $8.3912(12)$ & $54911.7856154(16)$ & $54911.78046975(81)$ \\
\hline U2 & EPIC-MOS1/FF thin & $8.39097(26)$ & $8.39136(29)$ & $54911.7440458(30)$ & $54911.7440479(17)$ \\
\hline $\mathrm{U} 2$ & EPIC-MOS2/FF thin & $8.39103(25)$ & $8.39118(35)$ & $54911.7440524(13)$ & $54911.7440483(15)$ \\
\hline $10701^{12}$ & HRC-S/LETG & & $8.39119(20)$ & $55085.6684206(14)$ & $55085.6684187(15)$ \\
\hline 1792 S3 & EPIC-pn/FF thin & $8.39102(12)$ & $8.39101(15)$ & $55096.30446151(69)$ & $55096.3044632(11)$ \\
\hline $\mathrm{S} 1$ & EPIC-MOS1/SW thin & $8.39097(20)$ & $8.39120(20)$ & $55096.2966932(13)$ & $55096.2966943(13)$ \\
\hline S2 & EPIC-MOS2/SW thin & $8.39104(15)$ & $8.39102(24)$ & $55096.2967896(14)$ & $55096.2967885(11)$ \\
\hline
\end{tabular}

Notes. The individual periods for ROSAT and Chandra HRC were not divided into soft (120-400 eV) and hard (400-1000 eV) band (see Sect. 3), hence we list the individual periods derived from all counts. We list the TOAs (definition equal to that in KvK05 and vK07) of the soft band (for the "all data" solution of the soft band, see Table 3) and of the hard band (for the "all data" solution of the hard band, see Table 3). The errors of the periods (Ransom et al. 2002) and the TOAs (derived from the fitted light curves, see text) are given in parenthesis and denote $1 \sigma$ confidence level. 